\title{
Characterisation of full-field deformation behaviour using digital imaging techniques
}

\author{
Y. H. Tai, M. Zanganeh, D. Asquith and J. R. Yates \\ Department of Mechanical Engineering, University of Sheffield, \\ Mappin Street, Sheffield, S1 3JD, UK. \\ y.tai@sheffield.ac.uk
}

Keywords: full-field measurements, diametral contraction, DIC, edge detection

\begin{abstract}
Optical techniques for displacement measurements have become more common in recent years. The current preferred technique is digital image correlation (DIC) which works very well but has limitations for measuring diametral contractions in cylindrical specimens using a standard 3D system. To overcome the limitations of using either a diametral clip gauge or standard 3D DIC, a method has been developed for measuring diametral contractions simultaneously in two directions using a standard 3D DIC system in conjunction with an edge detection algorithm. Results have shown the method to work well.
\end{abstract}

\section{Introduction}

Optical techniques for full-field measurements have been gaining popularity in the recent years especially with advances in digital imaging and computational capabilities. One method which has seen significant increase in use and capabilities is digital image correlation (DIC). It works very well for both 2D and 3D full-field measurements given unlimited resources. However, for a standard 3D DIC system with a pair of cameras, the measurement area for a cylindrical specimen is limited to approximately one third of the circumference. This is due to the maximum overlap area between any given pair of images.

Accurate measurement of the diametral contraction of cylindrical tensile specimens with various notch geometries are used in the development of local damage models of fracture. Due to the anisotropic material properties encountered in many structural metals, the diametral contraction should be measured in at least in the $x$ and $z$ directions (please refer to Figure 3 for illustration). A 3D DIC system can provide these measurements but the tests have to be repeated using different specimens.

This paper describes a technique developed based on edge detection used in conjunction with a standard Vic3D DIC system to obtain diametral contraction data in two orthogonal directions simultaneously during a tensile test. Tensile tests were done on cylindrical specimens of an aluminium alloy with both plain and notched profiles.

\section{Experimental work}

\section{Material}

The material used for this study was 2050-T851 aluminium lithium alloy in $15 \mathrm{~mm}$ thick plate form. Chemical composition of the plate is shown in Table 1 below. 
Table 1: Chemical composition of 2050-T851 alloy used (wt\%)

\begin{tabular}{|c|c|c|c|c|c|c|c|c|}
\hline $\mathrm{Ag}$ & $\mathrm{Zr}$ & $\mathrm{Mn}$ & $\mathrm{Cu}$ & $\mathrm{Si}$ & $\mathrm{Mg}$ & $\mathrm{Li}$ & $\mathrm{Zn}$ & $\mathrm{Al}$ \\
\hline 0.36 & 0.09 & 0.38 & 3.51 & 0.03 & 0.40 & 0.91 & 0.02 & Balance \\
\hline
\end{tabular}

\section{Specimen geometry}

Three different geometries of tensile specimens were used to allow for different states of triaxiality required for tuning the damage models. Specimen geometry G1 was a standard plain smooth tensile specimen. Specimen geometry G2 and G3 were notched with two different radii. Figure 1 illustrates the design and dimensions of the three specimens used which were machined from the longitudinal (L), transverse (T) and $45^{\circ}$ directions. Table 2 summarises the specifications and number of specimens used in this work. Further information on the design of the specimens can be found in literature [1]

Table 2: List of specimens and specifications

\begin{tabular}{|c|c|c|c|c|c|c|}
\hline Specimen type & No of Specimens & Gauge Length $(\mathrm{mm})$ & $\mathrm{D}(\mathrm{mm})$ & $R_{o}(\mathrm{~mm})$ & $\rho_{o}(\mathrm{~mm})$ & $\mathrm{R}_{\mathrm{o}} / \rho_{\mathrm{o}}$ \\
\hline $\mathrm{G} 1-\mathrm{L}$ & 2 & 40 & 10 & - & - & - \\
\hline $\mathrm{G} 2-\mathrm{L}$ & 2 & 40 & 10 & 4 & 6 & 0.7 \\
\hline $\mathrm{G} 3-\mathrm{L}$ & 2 & 40 & 10 & 3 & 2 & 1.5 \\
\hline $\mathrm{G} 1-\mathrm{T}$ & 2 & 40 & 10 & - & - & - \\
\hline $\mathrm{G} 2-\mathrm{T}$ & 2 & 40 & 10 & 4 & 6 & 0.7 \\
\hline $\mathrm{G} 3-\mathrm{T}$ & 2 & 40 & 10 & 3 & 2 & 1.5 \\
\hline $\mathrm{G} 1-45$ & 2 & 40 & 10 & - & - & - \\
\hline $\mathrm{G} 2-45$ & 2 & 40 & 10 & 4 & 6 & 0.7 \\
\hline $\mathrm{G} 3-45$ & 2 & 40 & 10 & 3 & 2 & 1.5 \\
\hline
\end{tabular}
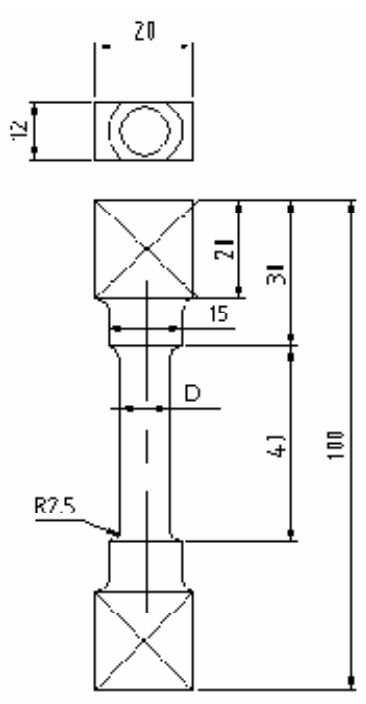
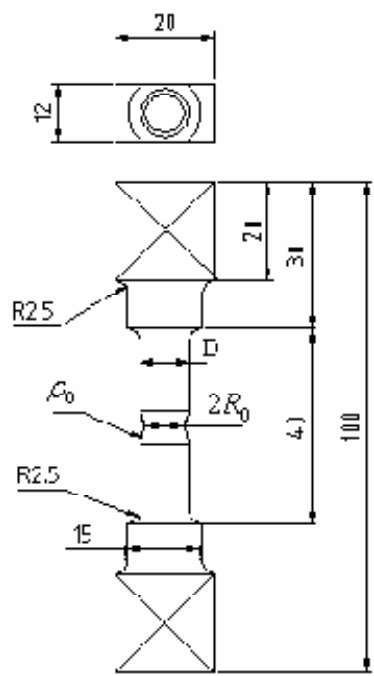
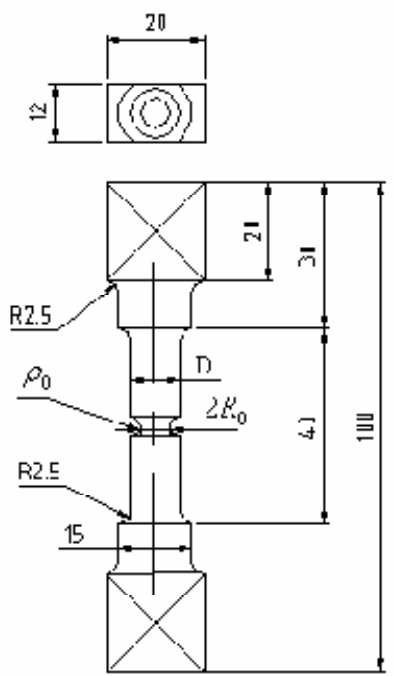

Figure 1: Specimen geometry G1, G2 and G3 (left to right) 


\section{Experimental set-up}

The primary aim of the experiments was to obtain diametral contraction measurements in both $x$ and $z$ directions as illustrated in

Figure 3. In previous work, this was done using a single axis diametral clip gauge which meant the tests had to be performed twice [1]. With the advances in digital imaging and measurement techniques, it was worth investigating the feasibility of using digital image techniques for measuring diametral contractions and preferably simultaneously in both directions.

For contraction in the $z$-direction, 3D DIC was used which in this case was a standard Vic3D system where two cameras were used to give a 3D perspective of the specimen surface. Figure 2 shows the images of a prepared specimen obtained by camera 1 and camera 2. Comparison of the two images shows that the overlap between two images is limited and hence the effective area available for deformation measurement is constrained to the area of overlap as shown in Figure 2. Unless the system is expanded with more cameras which incur significant cost, a standard 3D DIC system for diametral contraction measurements is effectively a substitute for a clip gauge albeit negating the need for repositioning when the position of the final necking is not known a priori.
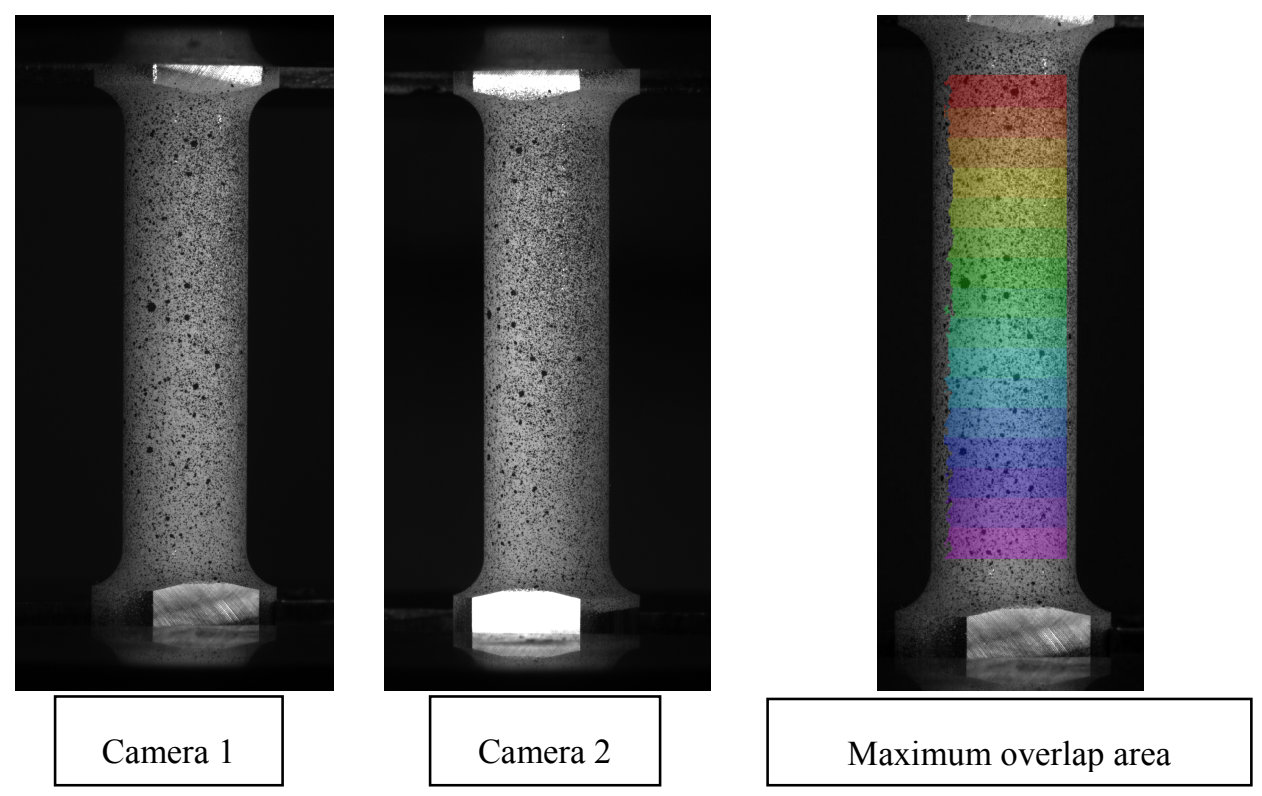

Figure 2: Illustration of images obtained with stereo set-up and resultant overlap area

To overcome the limitations of using a standard 3D system, a third digital camera (3) was positioned between the two cameras used for DIC. All three cameras had the same sensor types with a resolution of $5 \mathrm{MP}$.

Figure 3 illustrates the position of all three cameras with respect to specimen. The position of camera 3 allowed for diametral contraction in the $x$-direction to be measured by analyzing the digital images obtained using an edge finding routine which is described in the following section. Synchronisation of the images was done by matching the recorded load signals. The main advantage of whole set-up is that diametral contractions can be measured simultaneously on the same specimen in both $x$ and $z$ directions. The tests were carried out using an Instron 8501 servohydraulic machine fitted with hydraulic grips under displacement control at a constant rate of $0.01 \mathrm{~mm} / \mathrm{s}$ for all the tests. 


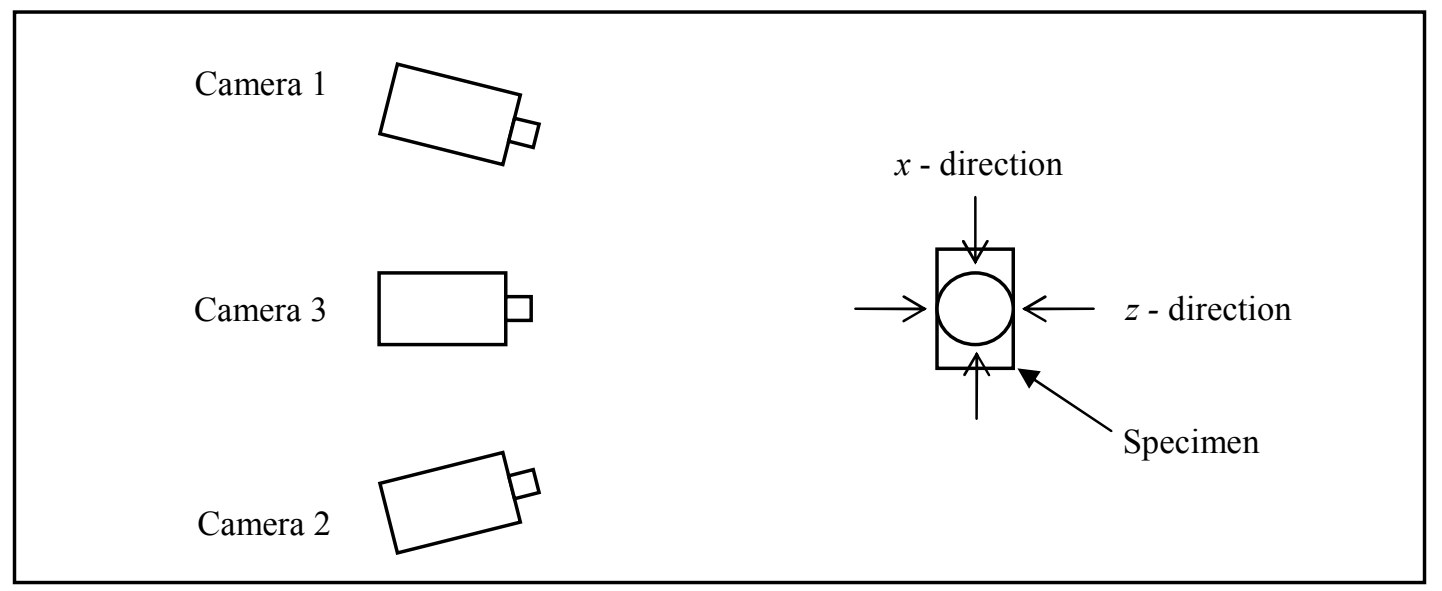

Figure 3: Plan view schematic of camera set-up w.r.t specimen

\section{Edge finding routine}

As shown in Figure 4, the edges of the specimen and consequently the load-diametral contraction of the specimens were measured using the Digital Image Processor 1.3 (DIP) [2]. The edge finding algorithm used in DIP is based on the extraction of the specimen from its background. The image obtained from camera 3 is an intensity image. To eliminate the background this intensity image needs to be converted to a binary image. This was carried out using a global threshold by minimizing the intraclass variance of the black and white pixels.

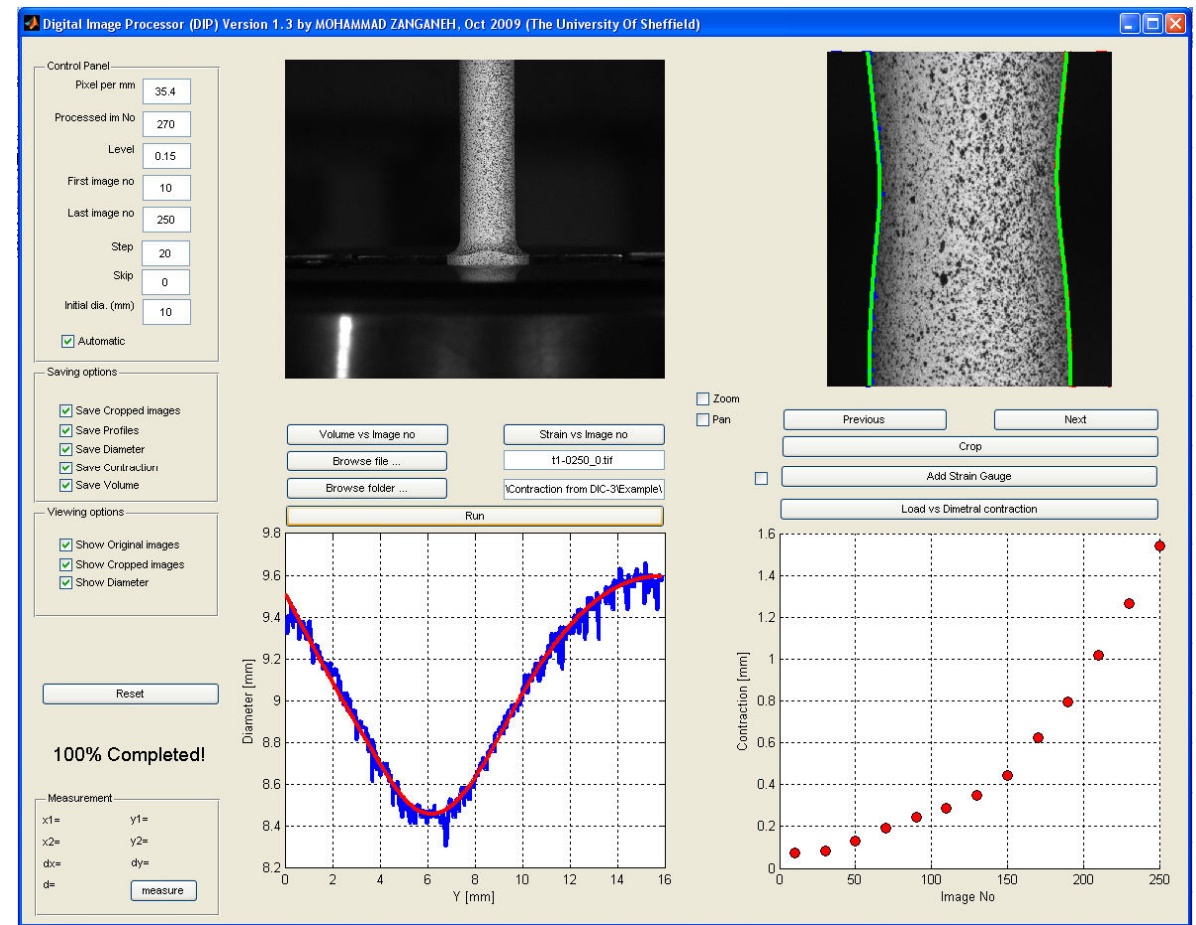

Figure 4: Digital Image Processor (DIP) user interface

Since the digital image correlation technique is being used simultaneously, a pattern of speckles is necessary on the surface of the specimen. Such speckles introduce some noise into the image. It was not possible to use back illumination for the $3^{\text {rd }}$ camera used for DIP to reduce the noise levels because it would interfere with the cameras used for DIC. The noise was eliminated by using a higher value for global threshold or a flood-fill operation based on morphological reconstruction. 
This would yield a matrix containing only zeros and ones where the zero elements are the background and the non-zero elements represent the specimen. However, the detected edges using above algorithm are still noisy, mainly because of required speckles on the specimen and hence the need for smoothing. To ensure that the outliners in the noisy data do not distort the behaviour of the smoothed curve, a locally weighted regression along with a robust smoothing procedure was used in DIP. A detailed explanation of this algorithm can be found in [2].

Apart from edge detection, DIP is capable of determining the Load vs Diametral contraction curve, true stress-strain curve, cross section area and volume of the specimen in the field of view. Furthermore, DIP can be used as a video extensometer in any direction using a two dimensional image correlation algorithm available in DIP V1.3.

\section{Results and discussion}

Images obtained from three cameras were processed using 3D DIC and DIP. Images from the first two cameras were correlated using Vic3D software. Since the eccentricity in loading is negligible and there is no buckling in the specimen (the loading condition is predominantly tension) the outof-plane displacement $(z$ - direction as shown in Figure 6$)$ is representative of the radial contraction of the specimen. On the other hand, the $x$ - direction diametral contraction can be measured using DIP as discussed in previous section. Experiments were performed in different material rolling directions with different triaxiality levels. Figure 5 to Figure 8 show the Load versus Diametral contraction determined using both techniques.

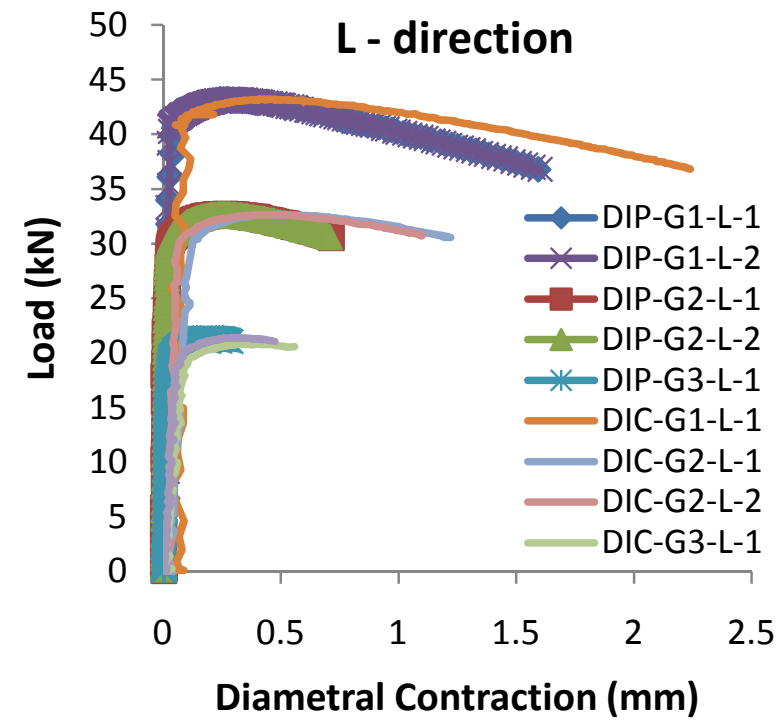

Figure 5: Load: Diametral contraction curve in L direction

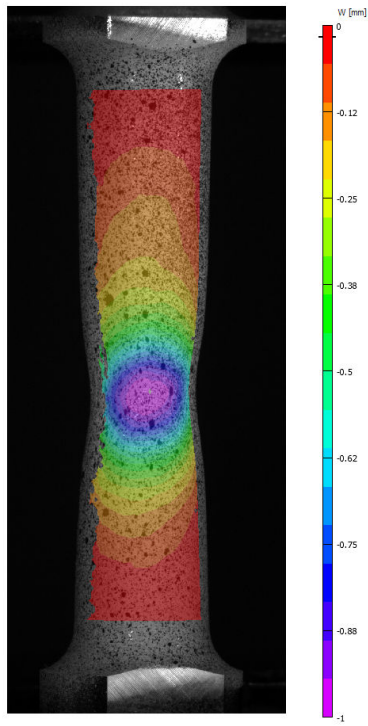

Figure 6: Out-of-plane deformation measured using DIC

Although, compared to DIP results, DIC results are slightly scattered in elastic region of loading, results obtained for different repetitions of the experiments are very consistent using either DIC or DIP. In plastic and post necking regions both techniques are able to represent the material behaviour very smoothly. As it is evident from Figure 7 and Figure 8 the load-diametral contraction curves determined for $\mathrm{T}$ and $45^{\circ}$ directions, using both techniques, matched well in most of triaxiality levels examined. However, there are some discrepancies in results obtained for L direction (Figure 5). This is mainly due to anisotropic contraction behaviour of this type of material. One the advantages of the technique explained is this paper is capability of measuring anisotropic behaviour.

The results also show that the edge detection technique is comparable to DIC for diametral contraction measurements. The maximum errors encountered in the technique were of the order of 3 pixels which in this particular case corresponded to 50 microns. 


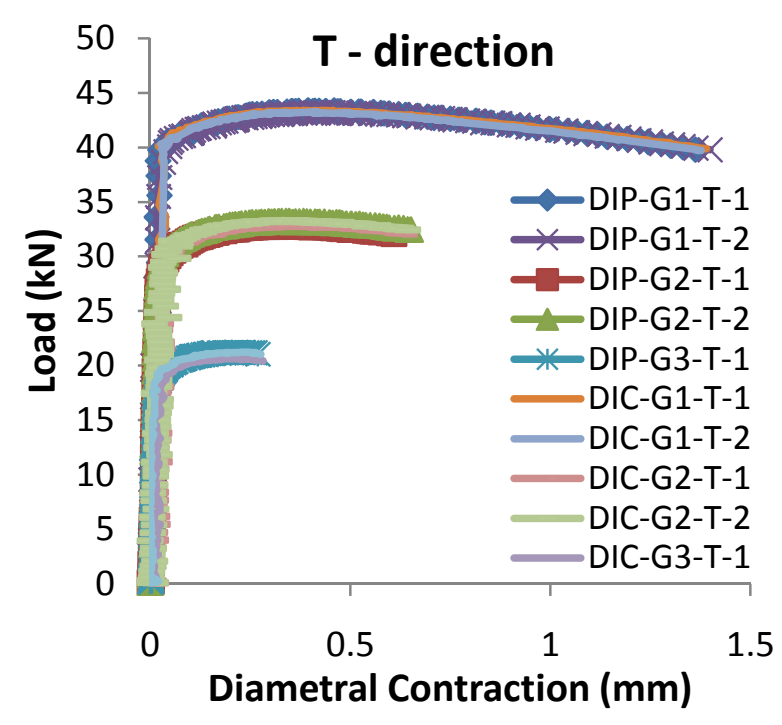

Figure 7: Load - Diametral contraction curve in $\mathrm{T}$ direction

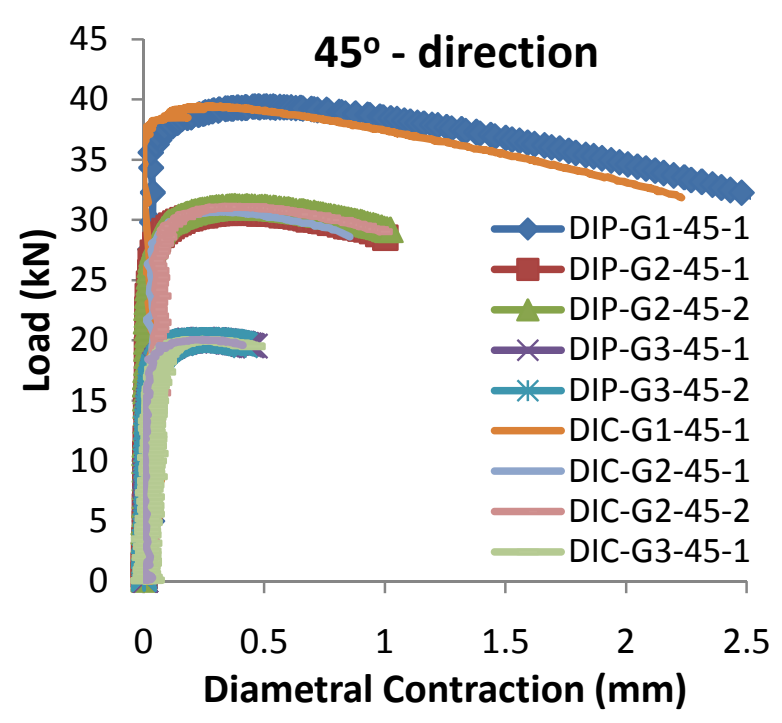

Figure 8: Load - Diametral contraction curve in $45^{\circ}$ direction

\section{Conclusion}

The combination of measuring diametral contraction by the edge detection technique and DIC has identified the extent of the deformation anisotropy in the 2050-T851 aluminium alloy used. Work is currently underway to better integrate the two techniques and generate $3 \mathrm{D}$ profiles of the surface of the specimen during deformation.

\section{References}

[1] Ayvar-Soberanis S, 3D CAFE modelling of ductile fracture in gas pipeline steel, (2008). Dept of Mechanical Engineering, University of Sheffield.

[2] Zanganeh M, Tai Y H, and Yates J R, An optical method of measuring anisotropic deformation and necking in material testing, (In press). 